GLASNIK MATEMATIČKI

Vol. 38(58)(2003), $285-298$

\title{
PERIODIC SOLUTIONS OF A FIRST ORDER DIFFERENTIAL EQUATION
}

\author{
LAVOSLAV ČAKLOVIĆ \\ University of Zagreb, Croatia
}

\begin{abstract}
The first order dynamical system $\dot{z}=F(t, z)$ is considered, where $F$ is $T$-periodic in time and sub-linear at infinity. Existence of $T$-periodic solution is proved, using degree theory, and applications to non-convex Hamiltonian systems is given as well.
\end{abstract}

\section{INTRODUCTION AND MAIN RESULTS}

General type equation. We consider the following first order differential equation

$$
\dot{z}=F(t, z)
$$

where $F \in C^{1}\left(\mathbb{R} \times \mathbb{R}^{N}, \mathbb{R}^{N}\right), N \in \mathbb{N}$, is $T$-periodic in time. Our goal is to find a $T$-periodic solution of (F) under the following sub-linearity condition at infinity

$$
\limsup _{|z| \rightarrow+\infty} \frac{|F(t, z)|}{|z|}=0 \text {, uniformly in } t,
$$

in Sobolev space $H_{T}^{1}:=\left\{z \in H^{1}\left(0, T ; \mathbb{R}^{N}\right) \mid z(0)=z(T)\right\}$ of $T$-periodic functions. A standard technique is to decompose the space in orthogonal sum

$$
H_{T}^{1}=E^{1} \oplus \mathbb{R}^{N}, z=u+m,
$$

where $E^{1}=\left\{u \in H^{1} \mid f u=0\right\}$, and $f z=\frac{1}{T} \int_{0}^{T} z(t) d t$ is the mean value of function $z(t)$. According to the above decomposition we split equation

2000 Mathematics Subject Classification. 34C25, 46N20, 47H15, 55M25, 58C30, $58 \mathrm{~F} 22$.

Key words and phrases. Differential equations, periodic solution, degree, Hamiltonian system. 
(F) to obtain $E^{1}$-component of $(\mathrm{F})$, an infinite dimensional equation, and $\mathbb{R}^{N}$-component of $(\mathrm{F})$, finite dimensional one:

$$
\begin{aligned}
\dot{u} & =F(t, u+m)-f F(t, u+m) \\
0 & =f F(t, u+m) .
\end{aligned}
$$

We would like to introduce a deformation parameter $\tau, 0 \leq \tau \leq 1$ to obtain a homotopicaly equivalent uncoupled system which has a solution. Following this idea we introduce

$$
\begin{aligned}
& \dot{u}=\tau[F(t, \tau u+m)-f F(t, \tau u+m)] \\
& 0=f F(t, \tau u+m),
\end{aligned}
$$

for $\tau=1\left(\mathrm{~F}_{\tau}\right)$ reduces to $\left(\mathrm{F}_{1}\right)$ and for $\tau=0$ we obtain an uncoupled system

$$
\begin{aligned}
& \dot{u}=0 \\
& 0=f F(t, m) .
\end{aligned}
$$

Solving $\left(\mathrm{F}_{\tau}\right)$ is equivalent to finding a zero of the function

$$
(m, u) \mapsto\left(f F(t, m+\tau u), u-\left(\frac{d}{d t}\right)^{-1}(\tau[F(t, m+\tau u)-f F(t, m+\tau u)])\right)
$$

defined on $\mathbb{R}^{N} \times E^{1}$. Some a priori bounds are needed and invertibility of $\frac{d}{d t}$ should be justified.

As it was kindly pointed out by the referee, it seems that instead the homotopy defined in formula $\left(\mathrm{F}_{\tau}\right)$ the homotopy in the proof of Theorem IV.3 in the Mawhin's book [9] can be used.

A priori bound. To obtain an a priori bound on the solution let us rewrite the sub-linearity condition (SL) in equivalent form:

$$
\begin{aligned}
& \forall \varepsilon>0, \exists C_{\varepsilon}>0, \text { such that } \\
& |F(t, z)| \leq \varepsilon|z|+C_{\varepsilon}, z \in \mathbb{R}^{N}, t \in \mathbb{R} .
\end{aligned}
$$

As shown in the next proposition some restrictions on $\varepsilon$ are essential for obtaining a priori bound on solution. See also an example in the proof of Theorem 1.3.

Proposition 1.1. Assume that $F$ is sub-linear at infinity, $m \in \mathbb{R}^{n}$ and $u \in E^{1}$ is a solution of the first equation in $\left(\mathrm{F}_{\tau}\right)$. If $\varepsilon$, in inequality $(\varepsilon S L)$, is such that $\varepsilon T<\sqrt{3} / 2$ in then

$$
\|u\|_{L^{\infty}} \leq \delta|m|+\gamma
$$

where

$$
\delta:=\frac{\varepsilon T}{\sqrt{T}-\varepsilon T} \text { and } \gamma:=\frac{C_{\varepsilon} T}{\sqrt{T}-\varepsilon T} .
$$


We prove the proposition in section 3 on page 291. Moreover, for any $r>0$ we introduce

$$
R_{\varepsilon}(r):=\frac{r+\gamma}{1-\delta}
$$

As we shall see in Lemma 3.1, inequality (1.1) implies that $z=m+u(t)$ is localized in the ball $B\left(|m|, R_{\varepsilon}(r)-r\right)$ whenever $|m| \geq R_{\varepsilon}(r)$.

There are two additional properties, we call them 'guiding function' and 'half space localization'. Each of them assures a priori bound on the solution.

\section{Guiding function.}

There exist a guiding function $W(z)$,

$$
\begin{aligned}
& W \in C^{1}\left(\mathbb{R}^{N}, \mathbb{R}\right), \text { and positive } r>0 \text { such that } \\
& |z| \geq r \Rightarrow F(t, z) \cdot W^{\prime}(z)>0 \text { uniformly on } t .
\end{aligned}
$$

As shown in Lemma 3.2, if $F$ has a guiding function then $|m| \geq R_{\varepsilon}(r)$ implies that the second equation $\left(\mathrm{F}_{\tau}\right)$ has no solution.

Half space localization.

There exists $r>0$ and a continuous

$$
\begin{aligned}
& \text { function } w: \mathbb{R}^{N} \backslash B\left(0, R_{\varepsilon}(r)\right) \rightarrow \mathbb{R}^{N} \text {, such that } \\
& |m| \geq R_{\varepsilon}(r) \Rightarrow(w(m) \cdot m \geq 0 \text { and } F(t, z) \cdot w(m)>0) \\
& \text { for all } z \in B\left(m, R_{\varepsilon}(r)-r\right) \text { uniformly on } t .
\end{aligned}
$$

If $z \in B\left(m, R_{\varepsilon}(r)-r\right)$ and $F$ satisfies half space localization property then $F(t, z)$ belongs to the half space $\left\{z \in \mathbb{R}^{N} \mid w(m) \cdot z>0\right\}$. Specially, this implies that the second equation $\left(\mathrm{F}_{\tau}\right)$ has no solution.

In both cases, i.e. if $F$ has guiding function or satisfies half space localization property, then, if there exists a solution $z=u+m$ of $\left(\mathrm{F}_{\tau}\right)$ it should satisfy $|m| \leq R_{\varepsilon}(r)$. Evidently, some additional property of $F$ is needed to prove the existence of solution. This is the non-triviality of degree as stated in next theorem.

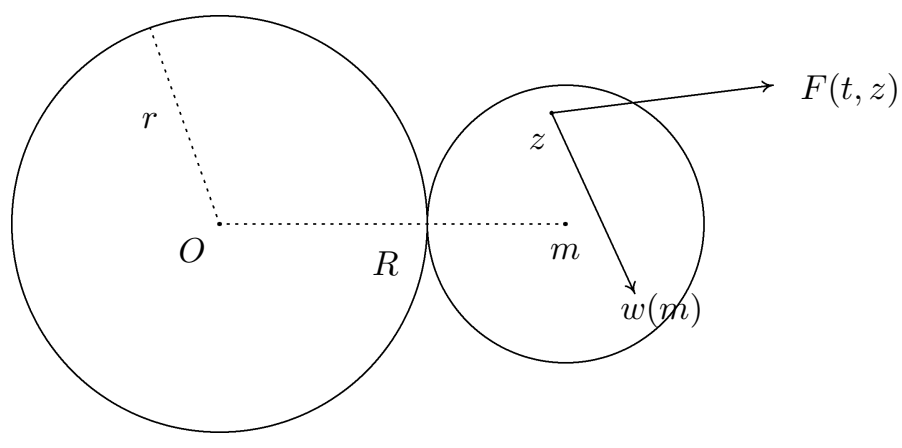

A priori bound 
TheOREM 1.2 (Krasnoselski). Assume that $F(t, z)$ satisfies (SL) (or $(\varepsilon \mathrm{SL}))$. If $F$ has guiding function and $d:=\operatorname{deg}\left(W^{\prime}, B(0, r), 0\right) \neq 0$ then (F) has a T-periodic solution.

A simple argument for introducing condition $\operatorname{deg}\left(W^{\prime}, B(0, r), 0\right) \neq 0$ is the situation when $F: \mathbb{R} \rightarrow \mathbb{R}$ is strictly positive. Then, equation $\dot{z}=F(z)$ has no periodic solutions, $\operatorname{deg}\left(W^{\prime}, B, 0\right)=0$ for any interval $B=(-r, r), r>0$ and condition (1.2) is fulfilled with $W^{\prime}=F$. Evidently, the degree $d$ has the same value for greater $r$ because of the non-vanishing derivative $W^{\prime}$ in (1.2).

Applications to some types of Hamiltonian systems are given in section 5. The theorem is a particular case of $[6$, Lema $6.5, \mathrm{Ch} .2]$ and we are not going to prove it here. Its proof is inspirative for more general statement in the next theorem.

THEOREM 1.3. Assume that $F(t, z)$ satisfies (SL) (or $(\varepsilon \mathrm{SL})$ ). If $F$ satisfies half space localization property and $\operatorname{deg}(w, 0, R) \neq 0$ then $(\mathrm{F})$ has a T-periodic solution.

An application to radial-like Hamiltonian is given in Theorem 1.4, with $w(m)=J m$.

Radial-like Hamiltonians. We consider the first order Hamiltonian system

$$
\dot{z}=J H^{\prime}(t, z)
$$

where $H(t, z): \mathbb{R} \times \mathbb{R}^{N} \rightarrow \mathbb{R}$ is $C^{1}$ function and $T$-periodic in time (prime denotes partial derivative with respect to $z$ ).

We say that Hamiltonian $H$ is strongly sub-quadratic at infinity if there exists $r>0$ and $1<p<2$ such that

$$
\left|H^{\prime}(t, z)\right| \leq \Theta_{2}|z|^{p-1} \text { whenewer }|z| \geq r .
$$

We say that Hamiltonian $H$ is radial-like if there exists $\mu>0$ such that

$$
\frac{H^{\prime}(t, z) \cdot z}{\left|H^{\prime}(t, z)\right||z|} \geq \mu>0 \text { for }|z| \geq r>0 \text { uniformly in } t \text {. }
$$

The following theorem is a consequence of Theorem 1.3.

THEOREM 1.4. Suppose that the Hamiltonian $H$ is radial-like an strongly sub-quadratic at infinity. Then:

i) $F=J H^{\prime}$ is sub-linear at infinity and satisfies (1.3) with $w(m)=J m$.

ii) Hamiltonian system $(H)$ has a T-periodic solution.

The same conclusion as in Theorem 1.4 can be proved using variational methods under additional hypothesis on Hamiltonian

$$
\left|H^{\prime}(t, z)\right| \cdot|z| \geq \beta \geq 0, \quad|z| \geq r \geq 0 \text { uniformly in } t .
$$

The proof can be found in [5].

A simple test for radial-like Hamiltonian is given in the following theorem. 
TheOREM 1.5. Assume that Hamiltonian $H$ is strongly sub-quadratic at infinity and satisfies

$$
\Theta_{1}|z|^{p} \leq H^{\prime}(t, z) z
$$

where $0<\Theta_{1} \leq \Theta_{2}$ and $1<p<2$. Then $H(t, z)$ is radial-like Hamiltonian.

Open problem. Is it possible to prove Theorem 1.4 under weaker condition

$$
\left.H^{\prime}(t, z) \cdot z>0, \quad|z| \geq r \geq 0 \text { ( uniformly in } t\right)
$$

instead of (Rad)?

Almost convex Hamiltonians. We also consider Hamiltonians that are weakly sub-quadratic in the sense

$$
\limsup _{|z| \rightarrow+\infty} \frac{|H(t, z)|}{|z|^{2}}=0, \quad \text { uniformly on } t .
$$

It seems that weak subquadraticity is not sufficient for existence of $T$-periodic solutions for $(\mathrm{H})$ even for radial-like Hamiltonians. We need an additional assumption

$$
H(t, z)=\hat{H}(t, z)-\frac{k}{2}|z|^{2}
$$

where $\hat{H}$ is strictly convex for some positive number $k$. Hamiltonian which satisfies (AC) we call almost convex. In other words, $H$ is almost convex if adding a quadratic term makes it strictly convex.

The following theorem is then easy to prove.

Theorem 1.6. Assume that the Hamiltonian $H(t, z)$ is radial-like, weakly sub-quadratic, and almost convex for $0<k<\frac{2}{T \sqrt{3}}$. Then, the Hamiltonian system $(\mathrm{H})$ has a T-periodic solution.

Evidently, without proof, we have the following corollary.

Corollary 1.7. Assume that Hamiltonian $H$ is radial-like, weakly subquadratic and convex. Then $(\mathrm{H})$ has a T-periodic solution.

\section{Some technical Results}

The framework for our problem is the space $H_{T}^{1}:=H^{1}\left(S_{T}, \mathbb{R}^{N}\right)$ of $T$ periodic functions from $\mathbb{R}$ to $\mathbb{R}^{N}$, here $S_{T}$ denotes the sphere $\mathbb{R} /[0, T]$, with a standard Hilbert space structure and norm

$$
\|z\|_{H^{1}}=\left(\int_{0}^{T}|z(t)|^{2} d t+\int_{0}^{T}|\dot{z}(t)|^{2} d t\right)^{1 / 2} .
$$

From now on we shall use shorthand notation $\int_{0}^{T} f$ for the integral $\int_{0}^{T} f(t) d t$. 
Lemma 2.1. For all $u \in E^{1}$ we have

$$
\|u\|_{L^{\infty}} \leq \sqrt{\frac{T}{12}}\|\dot{u}\|_{L^{2}} .
$$

Moreover, the constant $\sqrt{T / 12}$ is the best Sobolev constant in (2.1).

Proof. Let $u=\sum_{\substack{k \in \mathbb{Z} \\ k \neq 0}} u_{k} e^{i k \frac{2 \pi}{T} t}$ be Fourier expansion for $u$, where $u_{k} \in$ $\mathbb{R}^{N}$. Then

$$
\begin{aligned}
\dot{u}(t) & =\sum_{\substack{k \in \mathbb{Z} \\
k \neq 0}} i k \frac{2 \pi}{T} u_{k} e^{i k \frac{2 \pi}{T} t} \\
\|\dot{u}\|_{L^{2}} & =\frac{2 \pi}{T}\left(\sum_{\substack{k \in \mathbb{Z} \\
k \neq 0}} T k^{2}\left|u_{k}\right|^{2}\right)^{1 / 2} .
\end{aligned}
$$

On the other hand

$$
\begin{aligned}
|u(t)| & \leq \sum_{\substack{k \in \mathbb{Z} \\
k \neq 0}}\left|u_{k}\right| k \frac{1}{k} \leq\left(\sum_{\substack{k \in \mathbb{Z} \\
k \neq 0}}\left|u_{k}\right|^{2} k^{2}\right)^{1 / 2}\left(\sum_{\substack{k \in \mathcal{Z} \\
k \neq 0}} \frac{1}{k^{2}}\right)^{1 / 2} \\
& =\frac{\pi}{\sqrt{3}} \frac{\sqrt{T}}{2 \pi}\|\dot{u}\|_{L^{2}}=\sqrt{\frac{T}{12}}\|\dot{u}\|_{L^{2}} .
\end{aligned}
$$

This proves inequality. To see that $\sqrt{T / 12}$ is the best Sobolev constant in inequality (2.1) we take

$$
u=\sum_{k \neq 0} \frac{1}{k^{2}} e^{i k \frac{2 \pi}{T} t}
$$

with $\|u\|_{L^{\infty}}=u(0)=\sum_{k \neq 0} \frac{1}{k^{2}}=\frac{\pi^{2}}{3}$. On the other side,

$$
\|\dot{u}\|_{L^{2}}=\frac{2 \pi}{T}\left(\sum_{k \neq 0} T \frac{1}{k^{2}}\right)^{1 / 2}=\frac{2 \pi}{\sqrt{T}} \frac{\pi}{\sqrt{3}},
$$

which proves the claim.

The following lemma speaks about invertibility of $\frac{d}{d t}$.

LEMMA 2.2.

i) $L:=\frac{d}{d t}$ is a bounded linear operator from $H^{1}$ to $L^{2}:=L^{2}\left(S_{T} ; \mathbb{R}^{N}\right)$

ii) $L$ is bijective from $E^{1}$ onto $E=\left\{u \in L^{2} \mid f u=0\right\}$ and $L^{-1}: E \rightarrow E^{1}$ is an isomorphism of Banach spaces. 
iii) $L^{-1}: E \rightarrow E$ and $L^{-1}: E \rightarrow L^{\infty}:=L^{\infty}\left(0, T ; \mathbb{R}^{N}\right)$ are compact operators, and

$$
\left\|L^{-1}\right\|_{\mathcal{L}\left(E, L^{\infty}\right)} \leq \sqrt{\frac{T}{12}}
$$

Proof. i) follows from the definition of the norm on $H^{1}$. ii) Injectivity is clear: $N(L)=\left\{u \in E^{1} \mid \dot{u}=0\right\}=\{0\}$. To prove surjectivity let us take $v \in E$, i.e. $f v=0$. Then $z(t)=\int_{0}^{t} v(\tau) d \tau$ belongs to $H^{1}, \dot{z}=v$ and $z(t)$ is $T$-periodic. Put $z_{0}=z-f z$. Then $z_{0} \in E^{1}$ and $\dot{z}_{0}=v$.

That $L^{-1}: E \rightarrow E^{1}$ is an isomorphism follows from open mapping theorem.

iii) is a consequence of the well-known theorem of Rellich and Kondrachov (see H. Brezis [3]). The inequality follows from Lemma 2.1.

\section{A PRIORI BOUNDS}

Proof OF Proposition 1.1. Because $0 \leq \tau \leq 1$ it is sufficient to prove the proposition for $\tau=1$. Using inequality $\|u+m\|_{L^{\infty}} \leq\|u\|_{L^{\infty}}+|m|$ and inequality $(\varepsilon \mathrm{SL})$, one gets from $\left(\mathrm{F}_{\tau}\right)$, that

$$
\|\dot{u}\|_{L^{2}} \leq 2 \varepsilon T^{1 / 2}\left(\|u\|_{L^{\infty}}+|m|\right)+2 C_{\varepsilon} T^{1 / 2} .
$$

Using Lemma 2.1 we obtain

$$
2 \sqrt{3} T^{-1 / 2}\|u\|_{L^{\infty}} \leq 2 \varepsilon T^{1 / 2}\left(\|u\|_{L^{\infty}}+|m|\right)+2 C_{\varepsilon} T^{1 / 2}
$$

and finally

$$
\|u\|_{L^{\infty}} \leq \frac{\varepsilon T}{\sqrt{3}-\varepsilon T}|m|+\frac{C_{\varepsilon} T}{\sqrt{3}-\varepsilon T}=: \delta|m|+\gamma .
$$

which proves the inequality.

Lemma 3.1. Assume that $F$ is sub-linear at infinity. For a given $m \in \mathbb{R}^{N}$ let $u(t)$ is a solution of the first equation in $\left(F_{\tau}\right)$ and $r>0$. Then, for $|m| \geq R_{\varepsilon}(r)$ the following inequalities take place:

$$
|u(t)| \leq|m|-r \quad \text { and } \quad|u(t)+m| \geq r .
$$

Proof. i) Let us prove first that $|m|=R_{\varepsilon}$. Then, because of Proposition 1.1 ,

$$
\begin{aligned}
|u(t)| & \leq \delta \frac{r+\gamma}{1-\delta}+\gamma=\frac{\delta r+\gamma}{1-\delta}=\frac{r+\gamma}{1-\delta}-r \\
& =R_{\varepsilon}(r)-r=|m|-r
\end{aligned}
$$

ii) If $|m|=R>R_{\varepsilon}$, let us denote $r(R)=R(1-\delta)-\gamma$. Obviously $r(R)>r$ and, as above,

$$
|u(t)| \leq R-r(R) \leq|m|-r .
$$


To prove the second inequality in lemma let us calculate

$$
\begin{aligned}
|u(t)+m| & \geq|m|-|u(t)| \geq|m|-\delta|m|-\gamma \\
& =(1-\delta)|m|-\gamma \geq r .
\end{aligned}
$$

The following lemma is already proved in the book of Krasnoselski [6]. Because of its importance and simplicity we are giving a sketch of the proof.

Lemma 3.2 (Krasnoselski). Assume that function $F$ is sub-linear at infinity and has a guiding function for $|z| \geq r$. Then, equation $\left(F_{\tau}\right)$ has no solution with mean $m$ such that $|m| \geq R_{\varepsilon}(r)$.

Sketch of the Proof. Let $u \in E^{1}$ is a solution of the first equation $\left(\mathrm{F}_{\tau}\right)$. Then,

$$
0 \neq f F(t, \tau u(t)+m)
$$

Otherwise,

$$
\dot{u}=\tau F(t, \tau u+m)
$$

and for $z(t)=\tau u(t)+m$ we have

$$
\dot{z}=\dot{u}=F(t, z) .
$$

Using Lemma 3.1 and (1.2) and we finally have

$$
\frac{d}{d t} W(z(t))=\tau^{2} F(t, z(t)) \cdot W^{\prime}(z(t))>0
$$

which is impossible since $z$ is $T$-periodic.

\section{Proof of Theorem 1.3}

Let us consider a model Hamiltonian of the form

$$
H(z)=\frac{1}{p}|z|^{p}, \quad 1<p<2, \quad z \in \mathbb{R}^{2} .
$$

Using the fact that the corresponding energy is constant we can solve it explicitly using a substitution $z=r e^{i \phi(t)}$. But Theorem 1.2 cannot be applied. If we look carefully why this method fails, we see that the choice of function $W(z)$ is the cause of difficulties. The equation is

$$
\dot{z}=|z|^{p-2} J z .
$$

If we take $w(z)=J z$ and multiply both sides of the equation, we get

$$
\dot{z} \cdot J z=|z|^{p-2} J z \cdot J z=|z|^{p} .
$$

The right-hand side is positive, but the left-hand side cannot be written in the form $\frac{d}{d t} U(z)$ and we cannot prove an à priori bound on the solution. To overcome this difficulty we introduced half space localization property (1.3). 
Proof of theorem 1.3. Let us denote by $\varphi_{\tau}(u, m)$ a function from $E \times \mathbb{R}^{N}$ to $E$ defined by

$$
\varphi_{\tau}(u, m)=L^{-1}\{\tau[F(t, \tau u+m)-f F(t, \tau u+m)]\} .
$$

The function $\varphi_{\tau}$ is continuous in $(\tau, u, m)$ and compact. Solving $\left(\mathrm{F}_{\tau}\right)$ is equivalent to finding a zero of the function $\chi_{\tau}: E \times \mathbb{R}^{N} \rightarrow E \times \mathbb{R}^{N}$ defined by

$$
\chi_{\tau}(u, m)=\left(u-\varphi_{\tau}(u, m), f F(t, \tau u(t)+m) d t\right) .
$$

Because of inequality

$$
\|u\|_{L^{2}} \leq T^{1 / 2}\|u\|_{L^{\infty}} \leq T^{1 / 2}(\delta|m|+\gamma)
$$

it is more convenient to study solvability of equation $\chi_{\tau}(u, m)=0$ in the subset $\Omega=B_{1} \times B_{2}$ in $E \times \mathbb{R}^{n}$ where $B_{1}=B\left(0, \frac{\delta R+\gamma}{1-\delta} T^{1 / 2}\right) \subset E$ and $B_{2}=B(0, R) \subset \mathbb{R}^{N}$, with $R=\frac{r+\gamma}{1-\delta}$. To prove the existence of solution, it suffices to show that the degree

$$
d_{\tau}:=\operatorname{deg}\left(\chi_{\tau}, B_{1} \times B_{2},(0,0)\right), \quad \tau \in[0,1]
$$

is different from zero. Because of the half space localization property (1.3) and Lemma 3.1 the degree is well defined, because $f F(t, \tau u+m) \neq 0$ for $|m|=R$, and does not depend on $\tau$. We calculate it for $\tau=0$ :

$$
\begin{aligned}
d_{0} & =\operatorname{deg}\left(u \times f F(t, m), B_{1} \times B_{2},(0,0)\right) \\
& =\operatorname{deg}\left(i d_{E}, B_{1}, 0\right) \cdot \operatorname{deg}\left(f F(t, m), B_{2}, 0\right) \\
& =\operatorname{deg}\left(f F(t, m), B_{2}, 0\right)
\end{aligned}
$$

where we have used $\operatorname{deg}\left(i d_{E}, B_{1}, 0\right)=1$. Let us define $\bar{F}(m)=f F(t, m)$. Then $|m|=R$ implies that

$$
\bar{F}(m) \cdot w(m)=f F(t, m) \cdot w(m) d t>0 .
$$

We conclude that $\bar{F} / \partial B_{2}$ and $w / \partial B_{2}$ are homotopic and

$$
\operatorname{deg}\left(w, B_{2}, 0\right)=1 \neq 0 .
$$

This proves the theorem.

\section{Some consequences of Theorems 1.2 and 1.3}

Here are some examples of the first order Hamiltonian systems for which Theorem 1.2 is applicable.

Corollary 5.1. Suppose $F(t, z)=J H^{\prime}(t, z)$ sub-linear at infinity and

$$
H_{x}^{\prime} x-H_{y}^{\prime} y>0 \quad(\text { or }<0) \text {. }
$$

Then, the equation $(F)$ has a T-periodic solution. 
Here $W(z)=\frac{1}{2}\left(x^{2}-y^{2}\right)=\frac{1}{2} \operatorname{Re}|z|^{2}, w(z)=(x,-y)$ and $\operatorname{deg}\left(w, B_{R}, 0\right) \neq 0$ for $R>0$. In fact, $\operatorname{deg}\left(w, B_{R}, 0\right)=-1$ which is a consequence of excision and multiplicative properties of the degree.

Corollary 5.2. A special case of the previous corollary is the following one:

$$
H(t, z)=f(t)\left(\frac{1}{p}|x|^{p}-\frac{1}{p}|y|^{p}\right), \quad 1<p<2
$$

where $f(t)>0$ and is T-periodic. Then

$$
H_{x}^{\prime} x-H_{y}^{\prime} y=\left(|x|^{p}+|y|^{p}\right) f(t)>0
$$

and Corollary 5.1 can be applied.

Corollary 5.3. Let $H(t, z)=\frac{1}{p} f(t)|z|^{p}+g(t)|z|$ where $1<p<2$ and $f, g$ are real $T$-periodic continuous functions and $0<\alpha \leq \min f(t)$. Then the Hamiltonian system $\dot{z}=J H^{\prime}(t, z)$ has a T-periodic solution.

Proof. Indeed, $F(t, z):=J H^{\prime}(t, z)$ is sub-linear at infinity. It is sufficient to prove that $F$ satisfies the half space localization property. Let us define $w(m):=J m$ and choose $r>0$ such that

$$
\alpha r^{p-1}>\rho:=\max _{t \in[0, T]}|g(t)| \text {. }
$$

Then,

$$
w \cdot m=J m \cdot m=0
$$

and if $|m|=R_{\varepsilon}(r)=: R$ and $z \in B(m, R-r)$ then $|z|>r$ by Lemma 3.1 and

$$
\begin{aligned}
F(t, z) \cdot J m & =J H^{\prime}(t, z) \cdot J m=H^{\prime}(t, z) \cdot m \\
& =f(t)|z|^{p-2} z \cdot m+g(t) \frac{z}{|z|} \cdot m \\
& \geq \alpha r^{p-1} R-\rho R \geq\left(\alpha r^{p-1}-\rho\right) R>0
\end{aligned}
$$

which implies (1.3).

Corollary 5.4. Same conclusion as in Corollary 5.3 with hypothesis $H(t, z)=\frac{1}{p} f(t)|z|^{p}+g(t) z$ where $g:[0, T] \rightarrow \mathbb{R}^{N}$.

\section{RADiAL-Like HAMiltonians}

Proof of Theorem 1.4. It is sufficient to prove that for $|m|$ large enough, there exists $\varepsilon>0$ in $(\varepsilon \mathrm{SL})$, such that

$$
\sin \alpha:=\frac{R_{\varepsilon}(r)-r}{R_{\varepsilon}(r)}<\mu .
$$

In this case $H^{\prime}(t, z)$ is an element of the positive dual cone generated by the ball $B\left(m, R_{\varepsilon}(r)-r\right)$, hence an element of the half space $\left\{x \in \mathbb{R}^{2 N} \mid x \cdot m>0\right\}$ 


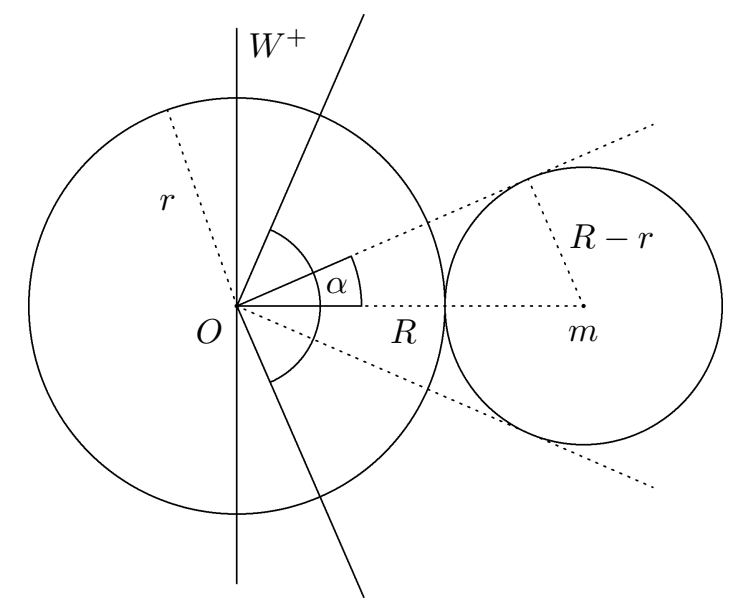

and obviously $f J H^{\prime}(t, z) \cdot J m=f H^{\prime}(t, z) \cdot m \neq 0$ which proves half space localization property. To prove (6.1) we have

$$
\frac{R_{\varepsilon}(r)-r}{R_{\varepsilon}(r)}=\frac{\frac{r+\gamma}{1-\delta}-r}{\frac{r+\gamma}{1-\delta}}=\frac{r \delta+\gamma}{r+\gamma}
$$

Then (6.1) is equivalent to

$$
r(\mu-\delta)>\gamma(1-\mu) .
$$

Now, $\varepsilon$ can be chosen such that $\mu-\delta>0$ and $r$ can be taken such that

$$
r>\frac{\gamma(1-\mu)}{\mu-\delta}
$$

which proves the theorem.

Proof of Theorem 1.5. Let $F(t, z)=J H^{\prime}(t, z)$. Evidently $F$ is sub-linear at infinity. To prove that $H$ is radial-like Hamiltonian let us consider $z$ such that $|z| \geq r$. Then

$$
\frac{H^{\prime}(t, z) \cdot z}{\left|H^{\prime}(t, z)\right||z|} \geq \frac{\Theta_{1}|z|^{p}}{\Theta_{2}|z|^{p}}=\frac{\Theta_{1}}{\Theta_{2}}=: \mu>0
$$

which proves the theorem.

\section{Almost convex Hamiltonians}

Proof of Theorem 1.6. The idea is to prove inequality (1.1) and to get a priori bound on the solution from this inequality. We shall write $(\mathrm{H})$ in the form

$$
L_{k} z:=\dot{z}+k J z=J \hat{H}^{\prime}(t, z)
$$

or, to simplify the notation

$$
L_{k} z=\hat{F}(t, z)
$$


with $\hat{F}(t, z)=J \hat{H}^{\prime}(t, z)$.

Let us perform decomposition of the equation (7.1) like in $\left(\mathrm{F}_{1}\right)$, i.e.

$$
\begin{aligned}
L_{k} u & =\hat{F}(t, u+m)-f \hat{F}(t, u+m) \\
0 & =f F(t, u+m) .
\end{aligned}
$$

The proof will be divided into several steps:

$1^{\text {st }}$ step: $\limsup _{|z| \rightarrow+\infty} \frac{|\hat{F}(t, z)|}{|z|} \leq 2 k$, uniformly on $t$.

$2^{\text {nd }}$ step: For any $u \in E$ such that $f u=0$ we have

$$
\|u\|_{L^{\infty}} \leq \frac{\sqrt{T}}{2 \sqrt{3}-k T}\left\|L_{k} u\right\|_{L^{2}}
$$

$3^{\text {rd }}$ step: If $u \in E, f u=0$, is a solution of the first equation (7.2) for given $m \in \mathbb{R}^{2 N}$, then

$$
\|u\|_{L^{\infty}} \leq \delta|m|+\gamma, \quad 0<\delta<1
$$

where $\delta=\frac{2(2 k+\varepsilon) T}{\sqrt{3}-5 k T-\varepsilon T}, \gamma=\frac{2 C_{\varepsilon} T}{\sqrt{3}-5 k T-\varepsilon T}$.

$4^{\text {th }}$ step: (Conclusion) Using (Rad) and the $3^{\text {rd }}$ step we obtain a priori bound on the solution, because (1.3) is satisfied with $w(m)=J m$.

Proof of the $1^{\text {st }}$ step, Let us denote by $\hat{G}$ the Legendre transform of $\hat{H}$, i.e.

$$
\hat{G}(t, v)=-\hat{H}(t, z)+v z
$$

where $v=\hat{H}^{\prime}(t, z)$ and $z=\hat{G}^{\prime}(t, v)$. Because of (WS) and the properties of the Legendre transform for each $\varepsilon, 0<\varepsilon<k$, there exists $C_{\varepsilon} \in \mathbb{R}$ such that

$$
\begin{aligned}
\frac{k-\varepsilon}{2}|z|^{2}-C_{\varepsilon} & \leq \hat{H}(t, z) \leq \frac{k+\varepsilon}{2}|z|^{2}+C_{\varepsilon} \\
\frac{1}{2(k+\varepsilon)}|v|^{2}-C_{\varepsilon} & \leq \hat{G}(t, v) \leq \frac{1}{2(k-\varepsilon)}|v|^{2}+C_{\varepsilon} .
\end{aligned}
$$

Functions $\hat{H}$ and $\hat{G}$ are bounded from bellow by a constant $-C_{\varepsilon}$ and consequently

$$
\begin{gathered}
\frac{1}{2(k+\varepsilon)}|v|^{2}-C_{\varepsilon} \leq \hat{G}(t, v) \leq v z-\hat{H}(t, z) \leq v z+C_{\varepsilon}, \\
\frac{k-\varepsilon}{2}|z|^{2}-C_{\varepsilon} \leq \hat{H}(t, z) \leq v z-\hat{G}(t, z) \leq v z+C_{\varepsilon} .
\end{gathered}
$$

Dividing (7.3) by $|v||z|$ we have

$$
\frac{1}{2(k+\varepsilon)} \frac{|v|}{|z|} \leq 1+\frac{2 C_{\varepsilon}}{|v||z|}, \quad \forall \varepsilon>0
$$

which proves the $1^{\text {st }}$ step. 
Proof of the $2^{\text {nd }}$ step.

$$
\begin{aligned}
\left\|L_{k} u\right\|_{L^{2}} & =\|\dot{u}+k J u\|_{L^{2}} \geq\|\dot{u}\|_{L^{2}}-k\|u\|_{L^{2}} \\
& \geq \frac{2 \sqrt{3}}{\sqrt{T}}\|u\|_{L^{\infty}}-k \sqrt{T}\|u\|_{L^{\infty}} \\
& =\frac{2 \sqrt{3}-k T}{\sqrt{T}}\|u\|_{L^{\infty}}
\end{aligned}
$$

where we have used inequality (2.1) from Lemma 2.1 and inequality $\|u\|_{L^{2}} \leq$ $\sqrt{T}\|u\|_{L^{\infty}}$.

Proof of the $3^{\text {rd }}$ step. Using the $1^{\text {st }}$ and $2^{\text {nd }}$ step in the first equation of $(7.2)$ we obtain

$$
\begin{aligned}
\frac{2 \sqrt{3}-k T}{\sqrt{T}}\|u\|_{L^{\infty}} & \leq\left\|L_{k} u\right\|_{L^{2}} \\
& \leq 2(2 k+\varepsilon) T^{1 / 2}\left(\|u\|_{L^{\infty}}+|m|\right)+2 C_{\varepsilon} T^{1 / 2}
\end{aligned}
$$

which proves the claim.

Corollary 7.1. Suppose that $H(t, z)=h(z)+g(t) z$, where $h(z)$ is a convex, radial-like and weakly sub-quadratic. If $g: \mathbb{R} \rightarrow \mathbb{R}^{N}$ is T-periodic and non-constant. Then, Hamiltonian system $H$ has a non-constant $T$-periodic solution.

\section{APPENDIX}

The following inequalities are useful in the theory of convex Hamiltonian systems. Let $G(z)=\gamma|z|^{q}+\alpha$ where $\gamma>0, q>1$ and $\alpha \in R$. Then if $\frac{1}{p}+\frac{1}{q}=1$,

$$
G^{*}(v)=\left(\frac{1}{\gamma q}\right)^{p / q} \frac{|v|^{p}}{p}-\alpha .
$$

If $H$ is a Legendre function, then:

$$
\begin{aligned}
& H(z) \leq \gamma|z|^{q}+\alpha \Longleftrightarrow H^{*}(v) \geq\left(\frac{1}{\gamma q}\right)^{p / q} \frac{|v|^{p}}{p}-\alpha, \\
& H(z) \geq \gamma|z|^{q}+\alpha \Longleftrightarrow H^{*}(v) \leq\left(\frac{1}{\gamma q}\right)^{p / q} \frac{|v|^{p}}{p}-\alpha .
\end{aligned}
$$

Proposition 8.1. Let $H$ be a Legendre function such that for some $\gamma>0$, $q>1, \alpha, \eta \in R$,

$$
\eta \leq H(z) \leq \gamma|z|^{q}+\alpha .
$$


If $\frac{1}{p}+\frac{1}{q}=1$ then

$$
\left(\frac{1}{\gamma q}\right)^{p / q} \frac{\left|H^{\prime}(z)\right|^{p}}{p} \leq H^{\prime}(z) z+\alpha-\eta
$$

Proof. Let $v=H^{\prime}(z)$. From (8.1) and (8.3) we obtain

$$
\left(\frac{1}{\gamma q}\right)^{p / q} \frac{|v|^{p}}{p}-\alpha \leq H^{*}(v)=v z-H(z) \leq v z-\eta .
$$

\section{REFERENCES}

[1] A. Bahri, H. Berestycki, Existence of forced oscillations for some nonlinear differential equations, Comm. Pure Appl. Math. 37 (1983), 403-442.

[2] H. Berestycki, Solutions périodiques de systèmes hamiltoniens, Séminaire Bourbaki, Soc. Math. France, Paris, 1983, 105-128.

[3] H. Brezis, Analyse fonctionelle, théorie et applications, Masson, Paris, 1983.

[4] F. Clarke, I. Ekeland, Hamiltonian trajectories having prescribed minimal period, Comm. Pure, Appl. Math. 33 (1980), 103-116.

[5] L. Čaklović, Periodic solutions of a first order nonconvex Hamiltonian system, Glas. Mat. 37 (2002), 101-118.

[6] M. A. Krasnosel'ski, The operator of translation along trajectories of ordinary differential equations, Mir, Moskow, 1966.

[7] M. A. Krasnosel'ski, Topological methods in the theory of nonlinear integral equations, Macmillan, New York, 1964.

[8] I. Ekeland, J. M. Lasry, On the number of Periodic trajectories for a Hamiltonian flow on a convex energy surface, Ann. Math. 112(1980), 283-319

[9] J. Mawhin, Topological degree methods in nonlinear boundary value problems, CBMS 40, Amer. Math. Soc., Providence, R.I., 1979.

[10] L. Nirenberg, Topics in nonlinear functional analysis, Lecture notes, Courant Institute of Math. Sc., New York Univ., 1974.

[11] P. H. Rabinowitz, Subharmonic solutions of Hamiltonian systems, Comm. Pure Appl. Math. 33 (1980), 609-633.

[12] P. H. Rabinowitz, Méthodes topologiques et problémes aux limits non linéaires, Lecture notes Univ. Paris VI, (1975), Redigées par H. Berestycki.

[13] M. Willem, Subharmonic oscillations of convex Hamiltonian systems, Nonlinear Anal. 9 (1985), 1303-1311.

L. Čaklović

Department of Mathematics

University of Zagreb

P.O.Box 335, 10002 Zagreb

Croatia

E-mail: caklovic@math.hr

Received: 20.06.2000.

Revised: 29.01.2003. 